\title{
A VALIAÇÃO DA CARGA DE TRABALHO FÍSICO E ANÁLISE BIOMECÂNICA DE TRABALHADORES DA CARBONIZAÇÃO EM FORNOS TIPO "RABO-QUENTE"1
}

Luciano José Minette ${ }^{2}$, Alexandre Santos Pimenta ${ }^{3}$, Manoel Marques de Faria ${ }^{4}$, Amaury Paulo de Souza Emília Pio da Silva ${ }^{4}$ e Nilton César Fiedler 6

\begin{abstract}
RESUMO - Este estudo teve como objetivo principal avaliar a carga de trabalho físico e a análise biomecânica de 20 trabalhadores da carbonização em fornos tipo "rabo-quente", localizados no Norte do Estado de Minas Gerais. Para análise da freqüência cardíaca, foi utilizado o sistema Polar Eletro Oy da Finlândia. O tempo consumido pelos trabalhadores por atividade foi avaliado através do método de cronometagem individual e a análise biomecânica, realizada por programa computacional desenvolvido pela Universidade de Michigan. Os resultados evidenciaram que a carga cardiovascular exigida para a realização da atividade era de $44,5 \%$ e classificada como moderadamente pesada. A operação que mais consumia tempo de trabalho era a vedação. Havia risco de compressão no disco vertebral $\mathrm{L}_{5}-\mathrm{S}_{1}$ na operação de carga, durante as fases de levantamento da rede e remoção de tora.
\end{abstract}

Palavras-chave: Ergonomia, frequiência cardíaca e lesão.

\section{EVALUATION OF THE PHYSICAL WORK LOAD AND BIOMECHANICAL ANALYSIS OF WORKERS AT WOOD CARBONIZATION IN HOT TAIL CHARCOAL KILNS}

\begin{abstract}
The main objective of this study was to evaluate the physical work load and the biomechanical analysis of 20 workers at the wood carbonization in hot tail charcoal kilns, located in the North of the Minas Gerais State, Brazil. Cardiac frequency was analyzed using the Polar Eletro Oy system from Finland. The time required by the workers per activity was evaluated through the individual chronometry method and the biomechanical analysis was carried out by a software developed by the Michigan University. The results showed that the cardiovascular load required was of $44.5 \%$, which was classified to be moderately heavy. The most time consuming activity was the sealing of the kiln. There is a compression risk of the $L_{5}-S_{1}$ vertebral disk for the kiln loading labor, during the steps basket lifting and log removal.
\end{abstract}

Keywords: Ergonomics, cardiac frequency and injury.

\footnotetext{
${ }^{1}$ Recebido em 19.09.2006 e aceito para publicação em 29.03.2007.

${ }^{2}$ Departamento de Engenharia Elétrica e de Produção da UFV. E-mail: <minetti@ufv.br>.

${ }^{3}$ BRICARBRAS - Briquetagem e Carbonização do Brasil Ltda. Jaguariaíva-PR. E-mail: <alexandrepimenta@bricarbras.com.br>.

${ }^{4}$ Programa de Pós-Graduação em Ciência Florestal da Universidade Federal de Viçosa (UFV).

${ }^{5}$ Departamento de Engenharia Florestal da UFV. E-mail: <amaurysouza@ufv.br>.

${ }^{6}$ Departamento de Engenharia Florestal, Centro de Ciências Agrárias da UFES, 29500-000 Alegre-ES. E-mail:<fiedler@pq.cnpq.br>.
} 


\section{INTRODUÇÃO}

A produção de carvão vegetal é uma atividade economicamente importante para o Brasil, destinada ao atendimento da demanda de diversos segmentos da indústria (siderurgia, metalurgia, cimento etc.), bem como para utilização residencial urbana e rural, com a ressalva de que um emprego direto na produção de carvão gera 8,32 empregos na economia como um todo (BRITO, 1999). Segundo a Associação Brasileira de Florestas Renováveis (2001), a produção de carvão vegetal em 1999 foi de 26,9 milhões de metros de carvão, tendo $70 \%$ desse valor sido obtido com madeira de reflorestamento. O carvão vegetal destinado ao uso siderúrgico representa uma das mais importantes atividades que alavancam o nosso desenvolvimento industrial, tendo visto que, em 1999, a produção de ferro-gusa foi de 24,5 milhões de toneladas.

Os fornos para fabricação de carvão do tipo "raboquente" são construídos utilizando-se apenas tijolos de barro cozido e argamassa de barro e areia. A argamassa utilizada é uma mistura de terra, areia e água em quantidades que produzam um barro fácil de trabalhar (ABRACAVE, 2001). Esses fornos são de baixo custo e fácil manuseio, uma vez que um carvoeiro pode operar de sete a 10 fornos, fazendo o trabalho de carga, carbonização, barrela, descarga e limpeza da praça. A jornada de trabalho inicia com o carregamento do forno de lenha e termina quando o carvão é descarregado e depositado no pátio.

A carbonização em fornos tipo "rabo-quente" apresenta riscos a saúde e segurança do trabalhador, podendo causar efeitos críticos ao organismo humano, como irritação ocular e nasal, dermatite, dores de cabeça, processo alérgico etc. A atividade de carbonização, principalmente as operações de carga e descarga, é considerada um trabalho pesado, pois transcorre em ambiente com temperatura elevada, causando danos à saúde do trabalhador, que no exercício de suas atividades adota posturas incorretas que podem ser lesivas à coluna vertebral durante o levantamento e a movimentação de cargas (LIMA e SOUZA, 2000).

Segundo o Ministério do Trabalho e do Emprego (BRASIL, 2003), é importante garantir condições de trabalho seguras e saudáveis, tendo como finalidade a preservação da saúde e da vida do trabalhador, prevenindo acidentes e doenças ocupacionais.
Minette (1996) citou que a ergonomia tem contribuído significativamente para a melhoria das condições de trabalho humano. Na carbonização de madeira, essa contribuição tem sido relativamente modesta, em virtude do baixo número de pesquisas e da pouca divulgação dos seus benefícios.

Na realização de estudos ergonômicos, é prática medir os índices fisiológicos com o objetivo de determinar o limite de atividade física que o indivíduo pode exercer. A aplicação da análise da carga cardiovascular através da freqüência cardíaca estabelece a carga de trabalho físico dentro dos limites que podem ser mantidos em uma jornada de trabalho de $8 \mathrm{~h}$ e a frequiência cardíaca não deve exceder a 110 bpm (COUTO, 1995).

Este estudo teve como objetivo principal avaliar a carga de trabalho físico e realizar avaliação biomecânica de trabalhadores envolvidos na carbonização em fornos do tipo "rabo-quente".

\section{MATERIAL E MÉTODOS}

\section{1. Área de estudo}

Os dados foram coletados em uma empresa florestal localizada no Norte do Estado de Minas Gerais, no período de 23 de março a 26 de junho de 2003.

\subsection{População e amostragem}

A coleta de dados foi realizada em uma amostra de 20 operadores, $100 \%$ da população considerada, que atuavam em fornos de carbonização do tipo "raboquente". Este trabalho teve como base a metodologia proposta por Apud (1989), Couto (1996) e Sant`Anna (1998).

\subsection{Descrição da atividade}

A área de trabalho era constituída por baterias de fornos do tipo "rabo-quente". A produção de carvão é simultânea em todos os fornos, e as diversas operações podem estar ocorrendo em momentos diferentes. A jornada de trabalho é de $8 \mathrm{~h}$ por dia, e durante esse tempo o mesmo trabalhador pode realizar as operações de carga, descarga, vedação, barrela e limpeza da praça, já que o processo de carbonização completo, do carregamento do forno a retirada do carvão, dura cerca de oito dias.

A operação de carga é caracterizada pela colocação da madeira no interior do forno, quando, em seguida, 
inicia-se a carbonização, ou seja, a queima da madeira, que dura geralmente três dias. Durante essa operação, o trabalhador supervisiona o processo, no mínimo, de hora em hora. A carbonização se contempla geralmente quando não mais se observar a saída de grande volume de fumaça pela chaminé do forno e a coloração da fumaça tender para azul transparente. É o momento indicado para a total vedação do forno e o início de seu resfriamento, sendo importante espargir barrela sobre o forno para facilitar a retirada do carvão. A descarga do forno então é iniciada com o auxílio de uma pá ou garfo. Após essa operação, realizam-se a limpeza e pequenos reparos nos fornos, para garantir boas condições na sua utilização.

\subsection{Avaliação da carga de trabalho}

Para a coleta e posterior análise dos dados de freqüência cardíaca dos trabalhadores de fornos de carbonização no decorrer da jornada de trabalho, foi utilizado um coletor de frequiência cardíaca da Polar Eletro Oy da Finlândia, modelo Polar Vantage NV. O aparelho, ajustado para armazenar os valores de frequiência cardíaca em intervalos de $5 \mathrm{seg}$ durante a jornada de trabalho, foi fixado ao trabalhador pela manhã, no início do serviço, e retirado à tarde, ao término da jornada.

Com os dados obtidos foi possível calcular a carga cardiovascular, conforme metodologia proposta por Apud (1989). A carga cardiovascular no trabalho corresponde à porcentagem da freqüência cardíaca durante o serviço em relação à freqüência cardíaca máxima utilizável, conforme expresso a seguir:

$$
\mathrm{CCV}=\frac{\mathrm{FCT}-\mathrm{FCR}}{\mathrm{FCM}-\mathrm{FCR}} \cdot 100
$$

em que:

$$
\begin{aligned}
& \text { CCV = capacidade cardiovascular, em \%; } \\
& \text { FCT }=\text { freqüência cardíaca do trabalho }(\mathrm{bpm}) ; \\
& \mathrm{FCM}=\text { freqüência cardíaca máxima }(220-\text { idade }) ; \\
& \mathrm{FCR}=\text { freqüência cardíaca repouso }(\mathrm{bpm}) ; \mathrm{e} \\
& \mathrm{FCL}=\text { freqüência cardíaca limite }(\mathrm{bpm}) .
\end{aligned}
$$

A freqüência cardíaca limite (FCL) (bpm) corresponde a $40 \%$ da carga cardiovascular do trabalho e é obtida pela seguinte fórmula.

$$
\mathrm{FCL}=0,40 \times(\mathrm{FCM}-\mathrm{FCR})+\mathrm{FCR}
$$

Quando a carga cardiovascular ultrapassar $40 \%$ (acima da freqüência cardíaca limite), será preciso reorganizar o trabalho, sendo determinado, de acordo com Apud (1989), o tempo de repouso (pausa) necessário, pela equação:

$$
T_{r}=\frac{H_{t}(F C T-F C L)}{F C T-F C R}
$$

em que:

$$
\begin{aligned}
& \mathrm{T}_{\mathrm{r}}=\text { tempo de repouso; } \mathrm{e} \\
& \mathrm{H}_{\mathrm{t}}=\text { tempo total de trabalho. }
\end{aligned}
$$

\subsection{Estudo do tempo consumido na atividade de carbonização}

Nesta avaliação foram utilizados dados obtidos a partir de uma amostra de 10 trabalhadores escolhidos por sorteio, por um dia, sendo seis trabalhadores avaliados nas operações de carga e descarga, dois na operação de limpeza e dois na operação de vedação. O resultado encontrado foi a média do tempo consumido em cada operação separadamente.

Foi utilizado o método de tempo individual, em que ocorre a detenção do cronômetro em cada ponto de medição, ou seja, cada operação foi medida individualmente ao longo da jornada (STÖHR, 1978).

\subsection{Análise biomecânica das operações de carbonização}

O estudo biomecânico foi concretizado por meio da análise bidimensional, utilizando-se a técnica de gravação em vídeotape, com o operador em diversos ângulos. Os movimentos foram "congelados" para medição dos ângulos dos diversos segmentos corpóreos. As cargas envolvidas foram medidas para aplicação do programa computacional de modelo biomecânico bidimensional de predição de posturas e forças estáticas, desenvolvido pela Universidade de Michigan, Estados Unidos. Foram fornecidos os ângulos das articulações obtidas durante a realização das operações (braços, tronco, coxofemorais, joelhos e tornozelos); o valor, a magnitude e a direção das forças envolvidas; mãos; e altura e peso do operador.

A análise por meio do software proporcionou encontrar a carga-limite recomendada, que corresponde ao peso que mais de $99 \%$ dos homens e $75 \%$ das mulheres conseguem levantar. A carga-limite recomendada induz

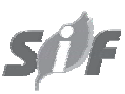

R. Árvore, Viçosa-MG, v.31, n.5, p.845-852, 2007 
a uma força (expressa em Newton) de compressão da ordem de $3.426,3 \mathrm{~N}$ sobre o disco $\mathrm{L}_{5}-\mathrm{S}_{1}$ da coluna vertebral, que pode ser tolerada pela maioria dos trabalhadores jovens e em boas condições de saúde.

\section{RESULTADO E DISCUSSÃO}

\subsection{Avaliação da carga de trabalho}

Para desenvolver a atividade de carbonização em fornos tipo "rabo-quente", a carga cardiovascular exigida é de 44,5\%, valor acima do recomendado por Apud (1989), que é de 40\%. A carga cardiovascular é um bom indicador da exigência física imposta pela atividade, 4, uma elevada carga cardiovascular requer grande esforço físico do trabalhador para desempenhar a atividade. Durante a execução de atividades que requer grande esforço cardiovascular, o fluxo sangüíneo para o coração pode ser prejudicado, a ponto de ocorrer tonteira e vertigem, quando se reduz o fluxo sangüíneo para o cérebro (KATCH e MCARDLE, 1996).

A frequiência cardíaca média durante toda a atividade foi de $112 \mathrm{bpm}$, com a ressalva de que a freqüência cardíaca limite era de 122 bpm, sendo observados picos de até $133 \mathrm{bpm}$. O trabalhador quando exposto a essa situação pode ter a integridade de sua saúde comprometida, ficando suscetível as Dort's, estresse, cansaço mental e problemas cardiovasculares, dentre outras patologias. $\mathrm{O}$ trabalho que exige frequiências cardíacas elevadas pode aumentar o suprimento de açúcar no sangue, dando origem a diabetes (RIO e PIRES, 1999).

De acordo com o Ministério do Trabalho e Emprego (BRASIL, 2003), a atividade pode ser classificada como moderadamente pesada. Segundo Couto (1983), um dos resultados esperados quando o trabalhador é exigido acima do limite é a fadiga física, que pode se manifestar das seguintes maneiras; tendência a cãibras, dores musculares, lombalgias e tendinites; absenteísmo; tremores e erros que podem levar a acidentes; envelhecimento precoce; uso excessivo de álcool como fonte de energia e redução do ritmo e qualidade do trabalho.

Levando em consideração o esforço físico que a atividade de carbonização em fornos tipo rabo-quente requer do trabalhador e a jornada de trabalho instituída de $8 \mathrm{~h}$ por dia, em cada hora o funcionário deveria trabalhar 46 min e descansar $14 \mathrm{~min}$. As atividades que demandam exigência física dos trabalhadores e que estão associadas à pressão de produção podem gerar prejuízos à saúde (APUD et al., 1999). As pausas são necessárias para evitar a sobrecarga de trabalho quando se detectam excessos de carga física, por isso não se recomenda a organização do trabalho em metas, visto que o trabalhador muitas vezes ignora as limitações do seu organismo, procurando encerrar a tarefa o mais breve possível, ignorando os malefícios das pausas irregulares.

\subsection{Estudo do tempo consumido na atividade de carbonização}

A análise do tempo exigido para a realização da operação de cada operação separadamente na atividade estudada, ao decorrer da jornada diária, certificou que a operação de carga de cinco fornos consumiu $3 \mathrm{~h}$ e 40 min, já a descarga, 2 h e 30 min, em que o trabalhador passa um tempo considerável do trabalho realizando essas duas operações. De acordo com Dias et al., (2002), a retirada do carvão do forno é a operação mais crítica de todo o processo de carbonização, visto que o trabalhador fica exposto a altas temperaturas e aos gases originados da combustão, além dos riscos de acidentes, como queimaduras. Em algumas situações, para a manutenção da qualidade do produto, o carvão é retirado ainda aquecido, aumentando a sobrecarga térmica e o risco de queimaduras corporais.

Também ficou evidenciado que as operações de limpeza e vedação desses fornos consumiram $3 \mathrm{~h} \mathrm{e}$ 30 min cada uma. Essas duas operações exigem do trabalhador posturas forçadas e incorretas, o que lhe causa grande desgaste físico nas articulações, músculos e ligamentos. Durante a realização dessas atividades, seriam de extrema importância pausas organizadas, com realização de ginástica laboral.

\subsection{Análise biomecânica}

A atividade de carbonização em fornos do tipo "rabo-quente" apresenta problemas biomecânicos como as posturas forçadas e incorretas e a aplicação de força. A postura de trabalho adotada pelos trabalhadores de maneira geral não é a mais correta. A má postura pode contribuir para desperdício energético, fadiga, lombalgias e outras dores. No carvoejamento de madeira com baixo grau de mecanização, o trabalhador freqüentemente aplica forças para levantar, empurrar, puxar, jogar ou transportar a madeira e o carvão. 
Das operações avaliadas, as únicas que apresentaram risco de lesão articular foram à carga e descarga dos fornos. Na operação de carga, todas as articulações apresentam risco de danos, já na descarga apenas as articulações $L_{5}-S_{1}$, quadril, joelho e tornozelos. $\mathrm{O}$ trabalho que exige manuseio de cargas excessivas, ou seja, aplicação de força durante a execução da tarefa, pode provocar deformações ósseas, dores musculares, fraturas, tendinites, bursites, inflamação das bolsas articulares em qualquer articulação do corpo humano (MERINO, 1996).

No Quadro 1, apresenta-se o resumo da análise biomecânica dessas operações. Para cada uma das fases destas atividades são mostrados se as articulações apresentam ou não algum problema causado pela carga de trabalho. A sigla SRL representa "Sem Risco de Lesão nas Articulações", ou seja, mais de $99 \%$ dos trabalhadores conseguem suportar a carga imposta pela atividade sem risco para as articulações envolvidas, e a sigla CLR representa "Carga Limite Recomendada Ultrapassada", ou seja, menos de $99 \%$ dos trabalhadores conseguem suportar a carga imposta pela atividade sem risco para as articulações envolvidas.

Os resultados da análise evidenciaram que somente a operação de carga na fase de levantamento da rede e remoção de tora oferece risco de compressão do disco vertebral $\mathrm{L}_{5}-\mathrm{S}_{1}$ por forças de $3.871 \mathrm{~N}$ e $3.635 \mathrm{~N}$ respectivamente. De acordo com Kisner (1998), a compressão do disco vertebral pode resultar em hérnias discais e ciatalgias, consequentemente aumentando a incidência de doenças ocupacionais, o que pode gerar absenteísmo e afastamentos prolongados.

Quadro 1 - Resumo da análise biomecânica das operações de carga e descarga do forno.

Table 1 -Summary of biomechanical analysis of the charcoal kiln loading and unloading activities

\begin{tabular}{|c|c|c|c|c|c|c|c|c|}
\hline \multirow[t]{2}{*}{ Operação } & \multirow[t]{2}{*}{$\begin{array}{c}\text { Fase da } \\
\text { operação }\end{array}$} & \multirow{2}{*}{$\begin{array}{c}\text { Postura } \\
\text { estática } \\
\text { selecionada } \\
\text { para análise }\end{array}$} & \multicolumn{6}{|c|}{$\begin{array}{l}\text { Articulações e suas respectivas condições de suportar a carga, } \\
\text { sendo Sem Risco de Lesão (SRL) e com risco de lesão, ou seja, } \\
\text { Carga Limite Recomendada Ultrapassada (CLR) }\end{array}$} \\
\hline & & & Ombros & Coto-velos & Disco L5/S1 & $\begin{array}{c}\text { Coxofe- } \\
\text { murais }\end{array}$ & Joelhos & Tornozelos \\
\hline Carga & Levantamento da tora & & SRL & SRL & CRL & CRL & SRL & SRL \\
\hline & Remoção da tora & & CRL & CRL & CRL & CRL & CRL & CRL \\
\hline & Enchimento da rede & & SRL & SRL & CRL & CRL & CRL & CRL \\
\hline & Levantamento da rede & $\frac{1}{100}$ & SRL & SRL & SRL & CRL & SRL & CRL \\
\hline
\end{tabular}

\section{CONCLUSÃO}

Ao término deste trabalho foi possível concluir que a atividade de carbonização em fornos do tipo "rabo-quente" requer esforço físico do trabalhador acima do limite recomendado da capacidade cardiovascular, sendo a atividade classificada como moderadamente pesada. As operações de carga e limpeza são as que consomem menor tempo, seguidas das de carga e vedação. Das operações avaliadas, somente as de carga e descarga podem provocar danos nas articulações dos trabalhadores. Além disso, existe um risco de compressão do disco vertebral $\mathrm{L}_{5}-\mathrm{S}_{1}$ na fase de levantamento da rede e remoção de tora durante a operação de carga.

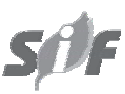

R. Árvore, Viçosa-MG, v.31, n.5, p.845-852, 2007 


\section{REFERÊNCIAS}

APUD, E. et al. Manual de ergonomia forestal. Concepción: Laboratório de ergonomia de la Universidad de Concepción, 1999.

APUD, E. Guidelines on ergonomics study in forestry. Genebra: ILO, 1989. $241 \mathrm{p}$.

\section{ASSOCIAÇÃO BRASILEIRA DE FLORESTA} RENOVÁ VEIS-ABRACAVE. Anuário

Estatístico. Disponível em: <http://

www.abracave.com.br>. Acesso em: 2001.

BRITO, J. O. Carvão vegetal no Brasil: gestões econômicas e ambientais. Revista de

Estudos. Avançados, v.4, n.9, p.221-227, 1999. Disponível em: <http://www.scielo.br/ scielo.php?script=sci_arttext\&pid=S01030141990000 200011\&lng=en\&nrm=iso $>$. Acesso em: 01/09/05.

COUTO, H. A. Ergonomia aplicada ao trabalho. O manual técnico da máquina humana. Belo Horizonte: Ergo, 1996. v.2. 383p.

COUTO, H. A. Ergonomia aplicada ao trabalho : o manual técnico da máquina humana. Belo Horizonte: Ergo, 1995. v.1. 353p.

COUTO, H. A. Fadiga física no

trabalho. Belo Horizonte: Ergo 1983. 42p.

(Cadernos Ergo, 5).

DIAS, E. C. et al. Processo de trabalho e saúde dos trabalhadores na produção artesanal de carvão vegetal em Minas Gerais. Cadernos de. Saúde Pública [online] v.18, p.269-277, 2002 Disponível: http://www.scielosp.org/ scielo.php?script $=$ sci_arttext $\&$ pid $=$ S0102$311 X 2002000100027 \& \operatorname{lng}=\mathrm{en} \& n r m=$ iso. Acesso em 17/09/06

KATCH, F.I.; MCARDLE, W. D. Nutrição, exercício e saúde. Rio de Janeiro: MEDSI, AED, 1996. 666p.

R. Árvore, Viçosa-MG, v.31, n.5, p.845-852, 2007
KISNER, C.; COLBY, L.A. Exercícios terapêuticos: fundamentos e técnicas. São Paulo: Manole, 1998.

LIMA, R. F.; SOUZA, R. C. R. Produção de carvão vegetal em pequena escala no Amazonas: aspectos legais e sócio-ambientais. In: ENCONTRO DE ENERGIA NO MEIO RURAL, 3, 2000, Campinas. Encontro...Campinas: 2000. p.1142-1152.

MERINO, E. A. D. Efeitos agudos e crônicos causados pelo manuseio e movimentação de cargas no trabalhador. 1996. 128f. Dissertação (Mestrado em Engenharia de Produção e Sistemas) - Universidade Federal de Santa Catarina, Florianópolis, 1996.

MinetTe, L. J. Análise de fatores operacionais e ergonômicos na operação de corte florestal com motosserra. 1996. 221f. Tese (Doutorado em Ciência Florestal) - Universidade Federal de Viçosa, Viçosa, MG, 1996.

BRASIL. Ministério do Trabalho e do Emprego.ASSUNTO 2003. Disponível em: www.mte.gov.br. Acesso em 03 mar. 2005.

RIO, R. P.; PIRES, L. Ergonomia: fundamentos da prática ergonômica. Belo Horizonte: Health, 1999. 225p.

SAnT'AnNA, C. M. Análise de fatores ergonômicos no corte de eucalipto com motosserra em região montanhosa.1998. 156f. Tese (Doutorado em Ciência Florestal) - Universidade Federal do Paraná, Curitiba, 1998.

STÖHR, G. W. D. Importância e aplicação do estudo do trabalho. In: CURSO DE ATUALIZAÇÃO SOBRE SISTEMAS DE EXPLORAÇÃOE TRANSPORTE FLORESTAL, 2, 1978, Curitiba. Anais... Curitiba: FUPEF, 1978. p.49-66 